\title{
IMPLICATIONS OF INERTIA FOR HYDROELASTIC INSTABILITY OF HERSCHEL-BULKLEY FLUIDS IN PLANE POISEUILLE FLOW
}

\author{
Shapour JAFARGHOLINEJAD \\ Department of Mechanical Engineering, Ardabil Branch, Islamic Azad University, Ardabil, Iran, and \\ Young Researchers and Elite Club, Ardabil Branch, Islamic Azad University, Ardabil, Iran \\ Mohammad Najafi \\ Department of Mechanical and Aerospace Engineering, Science and Research Branch, Islamic Azad University, Tehran, \\ Iran; e-mail:m.najafi.srbu@gmail.com
}

\begin{abstract}
This study investigates the effects of inertia on the hydroelastic instability of a pressure-driven Herschel-Bulkley fluid passing through a two-dimensional channel lined with a polymeric coating. The no-viscous hyperelastic polymeric coating is assumed to follow the two-constant Mooney-Rivlin model. In this work, analytical basic solutions are determined for both the polymeric gel and the fluid at very low Reynolds numbers. Next, the basic solutions are subjected to infinitesimally-small, normal-mode perturbations. After eliminating the nonlinear terms, two 4-th order differential equations are obtained. The equations with appropriate boundary conditions are then numerically solved using the shooting method. The results of the solution show that the inertia terms in the perturbed equations destabilize the pressure-driven Herschel-Bulkley fluid flow. The investigation reveals that the elastic parameter has a stabilizing effect on the flow. Also, based on the obtained results, the yield stress, depending on the power-law index, has a stabilizing or destabilizing effect on the flow. Since in this work the inertia terms are included in the pertinent governing equations, therefore, the results of this study are much more realistic and reliable than previous works in which inertia terms were absent. In addition, unlike the previous works, the present study considers both the shear-thinning and shear-thickening types of fluids. Hence, the results of this work embrace all the fluids which obey the Herschel-Bulkley model.
\end{abstract}

Keywords: inertia, instability, Herschel-Bulkley fluid, Mooney-Rivlin solid

\section{Introduction}

In general, most fluids flowing at very low Reynolds numbers, like creep flow, when passing through deformed channels sustain a type of instability called hydroelastic instability. This type of instability is much more intense when dealing with a non-Newtonian fluid passing through a channel coated with a highly-compliant material. In some industries, this instability phenomenon is of potential importance for enhancement of mixing in microfluidic devices. That is, due to vanishingly-small Reynolds numbers in such devices, they suffer from a very weak mixing because of the laminar nature of the creeping flow. To improve this poor mixing, the channel inner surface can be covered with a layer of a soft polymeric gel. By this way, when the fluid flows through the channel, certain waves may become unstable at the fluid-solid interface which eventually cause flow turbulence leading to a great improvement of the mixing performance which, in turn, advances the flow heat and mass transfer. The degree in improvement of the mixing, however, highly depends on the theological properties of both the solid gel and fluid. Basically, most of what is available in the literature with regard to the hydroelastic instability assumes Newtonian fluids, which is not realistic, at least, when dealing with mixing enhancement in microfluidic 
devices. In the previous years, the fluid viscous effects on hydroelastic instability in the creeping planar Poiseuille flow have been investigated by a few researchers in the field.

Kramer (1960) concentrated on advancing the concept of boundary layer stabilization, in particular, hydroelastic instability for many non-Newtonian fluids flowing through channels. In his works, he drew a conclusion that the stability of boundary layers could be affected by compliant coatings. In that sense, he was able to experience a considerable drag reduction for underwater moving bodies which were covered with some types of compliant coatings.

Gad-el-Hak (2002), Babenko and Kozlov (1972) and Fu and Joseph (1970) reported that although compliant materials can stabilize different types of instabilities, they might cause other types of instabilities such as hydro-and aero-elastic instabilities. In fact, it was noticed by researchers that stable fluid flows inside rigid channels became unstable in deformable channels.

Regarding fluid flows in microchannels, fluid mixing in such channels, in practice, is hardly possible according reports of Jensen (1999), Franjione and Ottino (1992) and Chien et al. (1986). In laminar flow domains, microscale dimensions lead to very low Reynolds numbers. Therefore, the concept of instability for such tiny devices becomes a prime concern when one is to advance the mixing effect in order to enhance the transfer of heat and mass as demonstrated by Kandlikar et al. (2005) and Ottino (1989).

Today, it is realized that rheological properties of both Newtonian and non-Newtonian fluids and coating materials used are very important for design purposes to reach fluid stability (Jafargholinejad, 2015; Muralikrishnan and Kumaran, 2002; Davies and Carpenter, 1997). Recently, Pourjafar et al. (2015) studied stability of power-law fluids in the creeping plane Poiseuille flow and analysed the effect of wall compliance on the stability of such fluids. In a study by Jafargholinejad et al. (2015), linear instability of viscoplastic fluids in the plane Poiseuille flow was investigated in the absence of inertia. Based on their results, the yield stress was found to have a stabilizing or a destabilizing effect on the pressure-driven flow of Herschel-Bulkley fluids, depending on the power-law exponent. However, Jafargholinejad et al. (2015) did not include the inertia terms and did not consider both the shear-thinning and shear-thickening fluids in their work.

Based on the above literature survey, to the best of the authors' knowledge, there is no published work on hydroelastic instability which takes into account the effects of inertia terms on both the shear-thinning and shear-thickening fluids.

The current study investigates the effect of inertia terms on the hydroelastic instability of pressure-driven Herschel-Buckley fluids flowing at very low Reynolds numbers through a two-dimensional channel lined with a polymeric solid gel. The no-viscous polymeric gel is assumed to follow the hyperelastic Mooney-Rivlin model since its solid model fits the rheological data for soft polymeric gels and provides more realistic results. In this study, very low laminar Reynolds numbers (creeping flow condition) are considered to simulate microfluidic fields industrial applications. Also, by varying the value of $n$, the power-law index, from $n<1.0$ to $n>1.0$, this study, unlike the previous works, considers the hydroelastic instability for both the shear-thinning as well as the shear-thickening fluids obeying the Herschel-Buckley non-Newtonian model.

\section{Physical description of the problem and governing equations}

Figure 1 illustrates a pressure-driven laminar, isothermal and viscoplastic fluid flowing (with no gravity effect) between two rigid parallel plates of $2 R$ height and $H R$ thickness coated with a compliant polymeric material. Both, the fluid and solid are assumed to have the same density and they are incompressible. The dashed curves in the figure illustrate a typical sinusoidal perturbation imposed on the fluid-solid interface. The origin of both the Lagrangian axes $(X, Y)$ and the Eulerian axes $(x, y)$ is set at the fluid-solid interface (Fig. 1). 


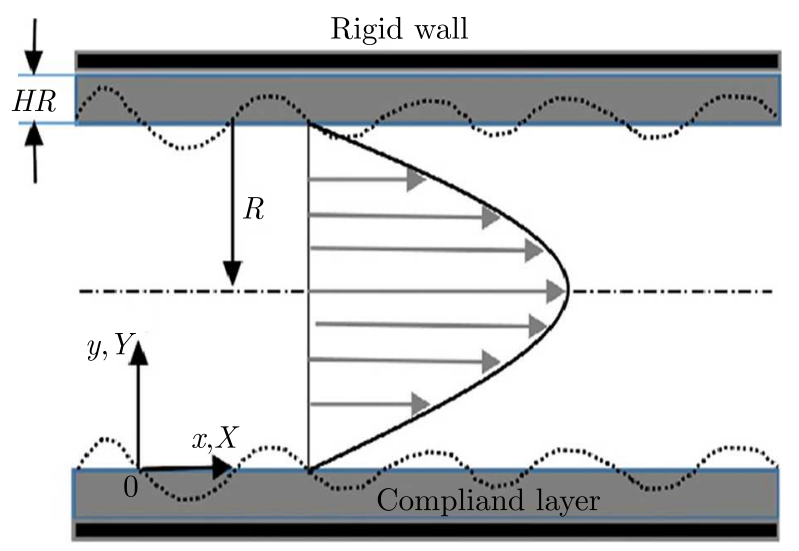

Fig. 1. Schematic of a channel lined with a compliant coating

The equations of motion for the solid are (Lai 2010)

$$
\operatorname{det}(\mathbf{F})=\left.1 \quad \rho_{s} \frac{\partial^{2} \mathbf{w}}{\partial t^{2}}\right|_{X}=\nabla_{X} \cdot \mathbf{P}
$$

In Eq. $(2.1)_{2}$, the subscript $X$ denotes the differentiation in the Lagrangian frame of reference, $\rho_{s}$ denotes density of the solid, $\mathbf{F}$ - deformation gradient tensor, $\mathbf{w}$ - current position of solid particles, and $\mathbf{P}$ is the Piola-Kirchhof stress tensor which is defined by

$$
\mathbf{P}=\boldsymbol{\sigma}\left(\mathbf{F}^{-1}\right)^{\mathrm{T}}
$$

where $\boldsymbol{\sigma}=-p \boldsymbol{\delta}+\boldsymbol{\tau}$ is the Cauchy stress tensor with $\boldsymbol{\tau}$ being the deviatoric part of the stress tensor. In dimensionless form, the stress tensor for the Mooney-Rivlin solid can be written as

$$
\boldsymbol{\sigma}=-p_{s} \boldsymbol{\delta}+\mathbf{B}+\frac{C_{2}}{C_{1}} \mathbf{B}^{-1}
$$

where $p$ is pressure, $\boldsymbol{\delta}$ - identity tensor, $\mathbf{B}=\mathbf{F F}^{\mathrm{T}}$ is the Cauchy Green strain tensor, and $C_{1}$ and $C_{2}$ are material constants (also known as the Mooney-Rivlin constants). Here, $C_{2} / C_{1}=\beta$, where $\beta$ is the elastic parameter. It needs to be mentioned that in this work $C_{1}$ (Mooney-Rivlin 1st parameter) is used for making all the stress terms dimensionless. Now, the momentum equation, in dimensionless form, for the solid side becomes

$$
\left.\operatorname{Re}_{s} \frac{\partial^{2} \mathbf{w}}{\partial t^{2}}\right|_{X}=\nabla_{X} \cdot \mathbf{P}
$$

where $\operatorname{Re}_{s}=\rho_{s}\left(C_{1} R^{2} / \eta^{2}\right)$ is referred to as the solid fictitious Reynolds number. Here, $\eta$ is the apparent viscosity of the fluid. It is noted here that $\eta / C_{1}$ is used to make 'time' dimensionless and $R$ is used for scaling the length.

For the fluid side, the conservation of mass equation is incorporated into the Cauchy equations of motion, and the resulting equations become (Lai et al., 2010)

$$
\rho_{f} \frac{D \mathbf{v}}{D t}=-\nabla p+\nabla \cdot \boldsymbol{\tau} \quad \nabla \cdot \mathbf{v}=0
$$

where $\rho_{f}$ is the fluid density and $D / D t$ is the material substantial derivative. Here, $\mathbf{v}$ denotes the velocity vector, $p$ is the isotropic pressure, and $\tau$ denotes the deviatoric stress tensor. In this 
study, it is assumed that the working fluid follows the Herschel Bulkley model; that is (Bird, 1987)

$$
\boldsymbol{\tau}_{f}=\left(T_{y}\left|\boldsymbol{\Pi}_{2 D}\right|^{\frac{-1}{2}}+m\left|\frac{1}{2} \boldsymbol{\Pi}_{2 D}\right|^{\frac{n-1}{2}}\right) 2 \mathbf{D}
$$

where $T_{y}$ denotes the yield stress, $\boldsymbol{\Pi}_{2 D}$ denotes the second invariant of the rate-of-deformation tensor, $m$ denotes the consistency index, $n$ is the power-law exponent, $2 \mathbf{D}=\nabla \mathbf{v}^{\mathrm{T}}+\nabla \mathbf{v}-$ rate-of-deformation tensor, and the superscript $\mathrm{T}$ denotes the velocity-gradient tensor $(\nabla \mathbf{v})$ transpose.

In dimensionless form, continuity equation $(2.5)_{2}$ remains unchanged, but the momentum equation $(2.5)_{1}$ becomes

$$
\operatorname{Re}_{f} \frac{D \mathbf{v}}{D t}=-\nabla p+\nabla \cdot \boldsymbol{\tau}
$$

where $\operatorname{Re}_{f}$ denotes the fluid Reynolds number which is defined as $\operatorname{Re}_{f}=\rho_{f}\left(C_{1} R^{2} / \eta^{2}\right)$.

Now, to find the base-state solution for the solid (assuming that both the velocity and shear stress are continuous at the fluid-solid interface), the following basic deformation and stress tensors in Lagrangian form are employed

$$
w_{X}(X, Y)=X+\frac{1}{1-\beta}\left[\frac{1}{2} G\left(Y^{2}-H^{2}\right)+\left(\tau_{y}-G\right)(Y+H)\right] \quad w_{Y}(Y)=Y
$$

where $G$ and $H$ are the pressure gradient and the coating thickness in dimensionless form, respectively. Therefore, the basic stress tensor for the solid becomes (Jafargholinejad et al., 2015)

$$
\boldsymbol{\sigma}=\left[\begin{array}{cc}
-p_{s}+1+\left(\frac{\partial w_{X}}{\partial Y}\right)^{2}+\beta & \frac{\partial w_{X}}{\partial Y}(1-\beta) \\
\frac{\partial w_{X}}{\partial Y}(1-\beta) & -p_{s}+1+\beta\left(1+\left(\frac{\partial w_{X}}{\partial Y}\right)^{2}\right)
\end{array}\right]
$$

Assuming that the fluid-solid interface is flat, then, in dimensionless form, the basic stress and basic velocity equations can be written as (Jafargholinejad et al., 2015)

$$
\boldsymbol{\sigma}=\left[\begin{array}{cc}
-p_{f} & \tau_{y}+\mu\left(\frac{\partial v_{x}}{\partial y}\right)^{n} \\
\tau_{y}+\mu\left(\frac{\partial v_{x}}{\partial y}\right)^{n} & -p_{f}
\end{array}\right] \quad v_{x}=-\frac{n}{n+1}\left(-\frac{G}{\mu}\right)^{\frac{1}{n}}\left[(1-y)^{\frac{n+1}{n}}-1\right]
$$

\section{Stability analysis}

Knowing the base-state solutions, they are now subjected to infinitesimally-small, time dependent, normal-mode perturbations in order to make linear stability analysis. Through the normalmode concepts (Drazin and Reid, 2004), the flow variables are expressed in terms of the sum of infinitesimal perturbation and the base-state solution, therefore

$$
f(x, y, t)=\bar{f}+f^{\prime}=\bar{f}+\widehat{f}(y) \mathrm{e}^{\mathrm{i} k x+\alpha t}
$$

where $\left(\bullet^{\prime}\right)$ denotes perturbation to the base-state, $(\boldsymbol{\bullet})$ is the perturbation amplitude, $(\bar{\bullet})$ denotes the basic flow, $k$ is the wave number of perturbation (a real number), $\alpha$ - the growth rate (a complex number), and $t$ - time.

To come up with a set of relations in terms of the perturbation variables, the perturbed variables are incorporated into the governing equations. After determining the appropriate boundary conditions and linearizing them, the intended set of equations are obtained. 
Now, based on what is described above, the solid and fluid side stability equations are derived. For the solid side, Eq. (3.1) is substituted into the appropriate governing equations for each field variable, and the resulting equations are linearized to obtain the following ordinary differential equation

$$
\begin{aligned}
& \operatorname{Re}_{s}\left\{\alpha^{2}\left[1+2 k-\mathrm{i} k^{2}\left(1+Q^{2}\right)+k d Q\right]\right\}+S_{0} w_{Y}(Y)+S_{1} d^{2} w_{Y}(Y) \\
& \quad+S_{2} d^{2} w_{Y}(Y)+S_{3} d^{3} w_{Y}(Y)+S_{4} d^{4} w_{Y}(Y)=0
\end{aligned}
$$

where

$$
\begin{aligned}
S_{0} & =\left(k^{2}+\mathrm{i} k^{2} \frac{\partial \bar{p}_{s}}{\partial Y}\right) d Q+\mathrm{i} k^{4} Q^{2}+\mathrm{i} k^{2} Q(d Q)-\mathrm{i} k^{4}-\mathrm{i} k^{2} \frac{\partial^{2} \bar{p}_{s}}{\partial Y^{2}} \\
& -k\left(d^{3} Q\right)+\beta\left[\mathrm{i} k^{4}\left(1+Q^{2}\right)-k^{3}(d Q)+2 \mathrm{i} k^{2}(d Q)^{2}+\mathrm{i} k^{2} Q\left(d^{2} Q\right)+k\left(d^{3} Q\right)\right] \\
S_{1} & =\left(2 k^{3}+2 \mathrm{i} k^{2} d Q\right) Q+k \frac{\partial^{2} \bar{p}_{s}}{\partial X \partial Y}-3 k(d Q)+\beta\left[3 k\left(d^{2} Q\right)-2 \mathrm{i} k^{2} Q(d Q)-2 k^{3} Q\right] \\
S_{2} & =-3 k d Q+2 \mathrm{i} k^{2}+\mathrm{i} k^{2} Q^{2}+\beta\left[-\mathrm{i} k^{2} Q^{2}+3 k(d Q)-2 \mathrm{i} k^{2}\right] \\
S_{3} & =2 k Q(\beta+1) \quad S_{4}=\mathrm{i}(\beta-1)
\end{aligned}
$$

where $Q=(1-\beta)^{-1}\left(G Y+\tau_{y}-G\right)$.

It is noted here that, in the special case when $\operatorname{Re}_{s}=0$, Eqs. (3.3) reduce to the analogous equation reported earlier by Jafargholinejad et al. (2015).

Now, for the fluid side, the linearized form of the second invariant of the rate-of-deformation tensor in the perturbed state can be written as

$$
\left(\Pi_{2 D}\right)^{\frac{n-1}{2}}=\lambda^{n-1}\left[1+\left((n-1) \frac{d \widehat{v}_{x}}{\lambda}+\mathrm{i}(n-1) k \frac{\widehat{v}_{y}}{\lambda}\right) \mathrm{e}^{\mathrm{i} k x+\alpha t]}\right.
$$

where $\lambda=d \bar{v}_{x} / d y$. The equations governing the perturbations for the fluid side can be shown as

$$
\mathrm{i} k \widehat{v}_{x}+d \widehat{v}_{y}=0
$$

and

$$
\begin{aligned}
\operatorname{Re}_{f} & {\left[\left(\frac{1}{\mathrm{i} k} \frac{d^{2} \widehat{v}_{y}}{d y^{2}}+\mathrm{i} k \widehat{v}_{y}\right) v_{x}(y)-\frac{1}{\mathrm{i} k} d \lambda+\alpha\left(\widehat{v}_{y}-\frac{1}{k^{2}}\right)\right]+F_{0} \widehat{v}_{y}+F_{1} d \widehat{v}_{y} } \\
& +F_{2} d^{2} \widehat{v}_{y}+F_{3} d^{3} \widehat{v}_{y}+F_{4} d^{4} \widehat{v}_{y}=0
\end{aligned}
$$

where

$$
\begin{aligned}
& F_{0}=\lambda^{n-1}\left(3 k^{2} \mu n^{2}-2 k^{2} \mu n-k^{2} \mu n^{3}\right)(d \lambda)^{2}+\lambda^{n}\left(k^{2} \mu n-k^{2} \mu n^{2}\right) d^{2} \lambda+\lambda^{n+1}\left(-k^{4} \mu n\right) \\
& F_{1}=\lambda^{n-1}\left[-\mu n k^{2}(n-1)(n-2)(d \lambda)^{2}\right]+\lambda^{n}\left[-\mu n k^{2}(n-1)(d \lambda)^{2}\right]+\lambda^{n+1}\left(-\mu n k^{4}\right) \\
& F_{2}=-4 k^{2} T_{y} d \lambda+\lambda^{n}\left(-2 k^{2} \mu n^{2}+6 k^{2} \mu n-4 k^{2} \mu\right) d \lambda \\
& F_{3}=-4 k^{2} T_{y} d \lambda+\lambda^{n}\left[-2 \mu k^{2}(n-1)(n-2) d \lambda\right] \\
& F_{4}=\lambda\left(4 k^{2} T_{y}\right)+\lambda^{n-1}\left(3 \mu n^{2}-2 \mu n-\mu n^{3}\right)(d \lambda)^{2}+\lambda^{n}\left(\mu n^{2}+\mu n\right) d^{2} \lambda+\lambda^{n+1}\left(4 k^{2} \mu-2 k^{2} \mu n\right)
\end{aligned}
$$

In the above equations, $d$ stands for the conventional total derivative $d / d y$. For Newtonian fluids (i.e., for $n=1$ ), this equation (Eqs. (3.7)) reduces to the Orr-Sommerfeld equation, as it should. In the special case of $n \neq 1$ and $\operatorname{Re}_{f}=0$, Eq. (3.7) reduces to the form reported by Jafargholinejad et al. (2015). 


\section{Boundary conditions}

To come up with right boundary conditions, the method of solution must be identified. In the present work, the Eulerian-Lagrangian type of framework is employed. That is, the Eulerian framework is used for the Herschel-Bulkley fluid variables, and the Lagrangian approach is used for the Mooney-Rivlin hyperelastic coating. Regarding the hyperelastic solid side, the no-slip condition is assumed at the channel inside walls. For the fluid side, however, the varicose mode perturbation is considered at the channel centreline. That is, the oscillation of the upper and lower fluid-solid interface goes out of phase, hence, based on Fig. 1, an even function of $y$ is assumed. Therefore, at the channel centreline the following boundary conditions can be used

$$
\left.\widehat{v}_{y}\right|_{y=1}=\left.0 \quad d \widehat{v}_{x}\right|_{y=1}=\left.0 \Rightarrow d^{2} \widehat{v}_{y}\right|_{y=1}=0
$$

with the assumption of no deformation for the outer side of the channel rigid wall

$$
\left.\widehat{w}_{Y}\right|_{Y=-H}=\left.0 \quad \widehat{w}_{X}\right|_{Y=-H}=\left.0 \Rightarrow d \widehat{w}_{Y}\right|_{Y=-H}=0
$$

For the Eulerian variables, keeping the first-order terms of the Taylor series expansion, the following conditions can be written

$$
\begin{aligned}
& \left.\Phi\right|_{\text {perturbed interface }}=\left.\Psi\right|_{\text {perturbed interface }} \\
& \left.\left.\Phi\right|_{y=0}+\left.\widehat{w}_{X} \frac{\partial \Phi}{\partial x}\right|_{y=0}+\left.\widehat{w}_{Y} \frac{\partial \Phi}{\partial y}\right|_{y=0}\right)=\left.\Psi\right|_{\text {perturbed interface }}
\end{aligned}
$$

where $\Phi$ and $\Psi$ refer to arbitrary variables at the interface for the fluid and solid sides, respectively.

At $y=Y=0$, the linearized form of the boundary conditions becomes

$$
\left.\widehat{v}_{y}\right|_{y=0}=\left.\left.\alpha \widehat{w}_{Y}\right|_{Y=0} \quad \widehat{v}_{x}\right|_{y=0}+\left.\lambda_{0} \widehat{w}_{Y}\right|_{Y=0}=\left.\alpha \widehat{w}_{X}\right|_{Y=0}
$$

and

$$
\begin{aligned}
& n \mu\left(\frac{d u_{0}}{d y}\right)^{n-1}\left[\frac{d \widehat{v}_{x}}{d y}+\mathrm{i} k \widehat{v}_{y}\right]+\widehat{w}_{Y} G+\mathrm{i} k \widehat{w}_{Y}\left(\frac{d f}{d y}\right)^{2}-\mathrm{i} k \widehat{w}_{Y}-\frac{d f}{d y} \frac{d \widehat{w}_{Y}}{d Y}-\frac{d \widehat{w}_{X}}{d Y} \\
& \quad+\beta\left(2 \mathrm{i} k \widehat{w}_{Y}+\frac{d f}{d y} \frac{d \widehat{w}_{Y}}{d Y}+\frac{d \widehat{w}_{X}}{d Y}\right)=0
\end{aligned}
$$

and

$$
\begin{array}{r}
-\widehat{p}_{f}+\widehat{p}_{s}-\mathrm{i} k \widehat{w}_{Y}\left[\mu\left(\frac{d u_{0}}{d y}\right)^{n}+\tau_{y}\right]+2 \mu\left(\frac{d u_{0}}{d y}\right)^{n-1} \frac{d \widehat{v}_{y}}{d y}+2 \tau_{y}\left(\frac{d u_{0}}{d y}\right)^{-1} \frac{d \widehat{v}_{y}}{d y} \\
+\mathrm{i} k \widehat{w}_{Y} \frac{d f}{d y}-2 \frac{d \widehat{w}_{Y}}{d Y}-k^{2} T \widehat{w}_{Y}+\beta\left(-2 \mathrm{i} k \widehat{w}_{Y}-2 \frac{d f}{d y} \frac{d \widehat{w}_{X}}{d Y}-\mathrm{i} k \widehat{w}_{Y} \frac{d f}{d y}\right)=0
\end{array}
$$

where $\lambda_{0}=\left.\left(d v_{x} / d y\right)\right|_{y=0}$ and $T=\gamma /\left(C_{1} R\right)$, and where $T$ is dimensionless interfacial tension and $\gamma$ is interfacial tension.

\section{Numerical method and results}

To make the stability analysis, Eqs. (3.3) for the solid side and Eqs. (3.7) for the fluid side and their pertinent boundary conditions are to be solved. To do so, a computer code is developed which uses the shooting method. A similar method like the one utilized in the present study, was 
used by Lee and Finlayson (1986) as well as Gkanis and Kumar (2005). It has also been used in the author's earlier works and produced reliable results even for the Mooney-Rivlin solids. The proposed code takes Eqs. (3.3) and (3.7) for the solid and fluid sides respectively together with the pertinent boundary conditions (Eqs. (4.1)-(4.7) as the input. The code begins solution at one end of the proposed boundary and shoots to the other end using an initial value solver iteratively to the point that the boundary condition at the other end is converged to its precise value. It has to be mentioned here that the developed code is verified for Newtonian data for a channel lined with a neo-Hookean gel under creeping flow conditions, see Gkanis and Kumar (2005) for details.

To solve Eqs. (3.3) and (3.7) simultaneously, two 4th-order boundary-value problems (Eqs. (3.3) and (3.7)) are treated like initial-value problems since the fluid and solid boundary conditions are coupled at the interface. Next, each 4th-order differential equation is transformed to a system of four 1st-order, linear and homogenous ordinary differential equations having a total of four unknown coefficients. To solve these ordinary differential equations, four boundary conditions are required for each, the fluid and the solid. Noting that there are only two explicit boundary conditions available (two for the fluid, Eqs. (4.1), and two for the solid, Eqs. (4.2)), the other two orthogonal boundary conditions have to be guessed for the fluid side and for the solid side to solve the problem. These unknown constants can be determined by constructing a matrix which contains the interfacial boundary conditions (Eqs. (4.5)-(4.7)). The computer code uses the developed shooting technique, takes the above mentioned matrix and other inputs like the Reynolds numbers, power-law index, surface tension, elastic parameter, and wall thickness, and by varying the channel pressure gradient, it calculates the real value of $\alpha$ and the growth rate of perturbations. Now, at the critical pressure gradient $\operatorname{real}(\alpha)=0$, the flow is stable for $\operatorname{real}(\alpha)<0$ and unstable flow is experienced for $\operatorname{real}(\alpha)>0$. Regarding the shooting technique, it actually uses the methods developed for solving initial value problems. The idea is to write the boundary value problem in vector from and to begin solution at one end of the boundary value problem and shoot to the other end with an initial value solver until the boundary condition at the other end converges to its precise value.

(a)

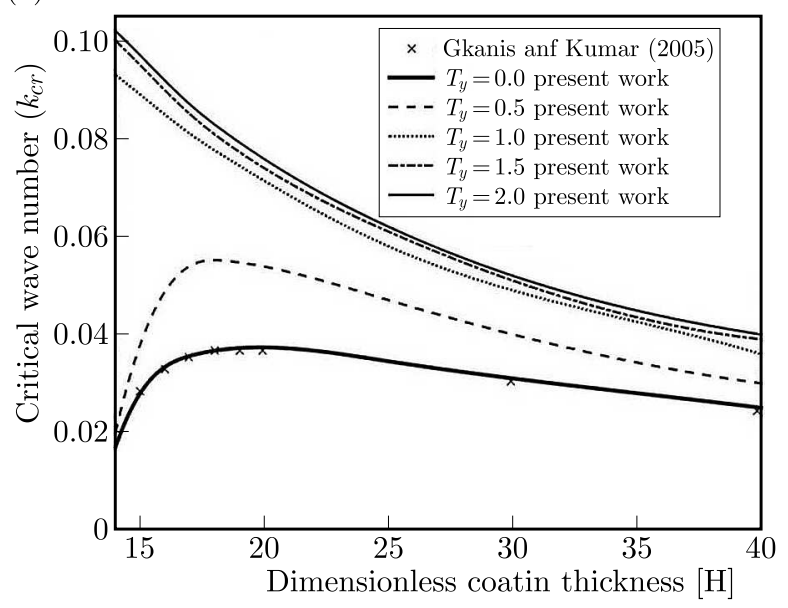

(b)

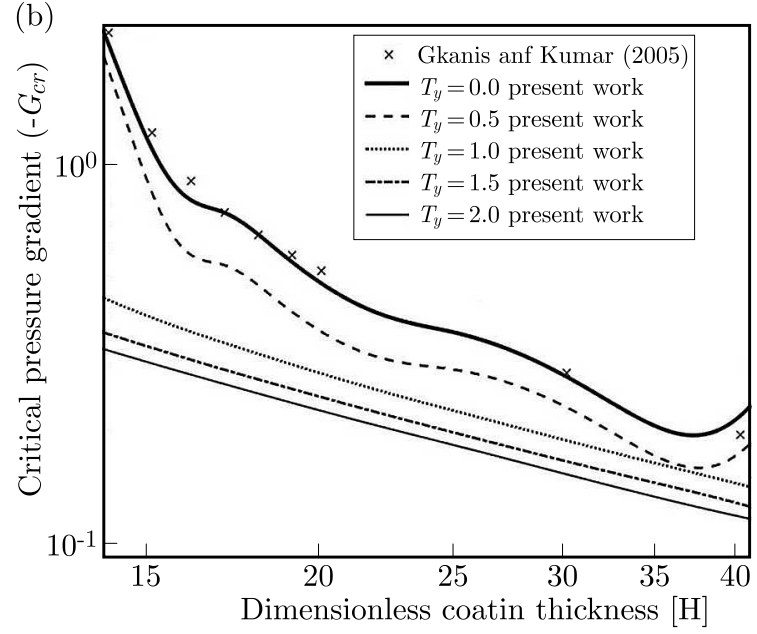

Fig. 2. Comparison between the present results for Newtonian fluids (solid line) and the numerical data reported by Gkanis and Kumar (2005), (symbols) at Re $=0$ for: (a) the critical pressure gradient, and

(b) the critical wave number

Figures $2 \mathrm{a}$ and $2 \mathrm{~b}$ illustrate the effects of the yield stress on the critical wave number and the critical pressure gradient at different compliant coating thicknesses, respectively. The figures also compare the plots of the present work obtained from the developed code for when the fluid becomes Newtonian (i.e. $n=1$ and $T_{y}=0$ in Eq. (2.6)) with the Newtonian data of Gkanis 
and Kumar (2005). This comparison actually verifies the present results as they coincide with the data of Gkanis and Kumar (2005) in both Figs. 2a and 2b. In Fig. 2a, as it can be seen, at any given coating thickness the critical wave number is increased as the yield stress increases, meaning that the fluid when at short wavelengths extracts energy from the flow and becomes unstable. Based on this figure, as the yield stress increases, the peak of the plot disappears, meaning that, unlike Newtonian fluids, for viscoplastic fluids, as the coating thickness increases, a monotonic drop in the critical wave number takes place. Again, unlike Newtonian fluids, at very small coating thicknesses, large wave numbers become unstable. Based on what can be seen in Fig. 2b, the yield stress actually destabilizes the flow. That is, the critical pressure gradient decreases as the fluid yield stress increases.

Figure 3 shows the variation of the critical pressure gradient versus the power-law index for very small Reynolds numbers. As it can be seen in this figure, an increase in the Reynolds number causes the fluid flow to be more unstable. In other words, the inertia terms have a destabilizing effect on the fluid flow under the creeping condition. Based on this figure, as the Reynolds number increases, the critical pressure gradient in the flow decreases which means the fluids become more and more unstable. Therefore, a better mixing process takes place in microchannels lined with flexible gels. However, at a given Reynolds number, this instability effect diminishes as a shear-thinning fluid approaches Newtonian behaviour.

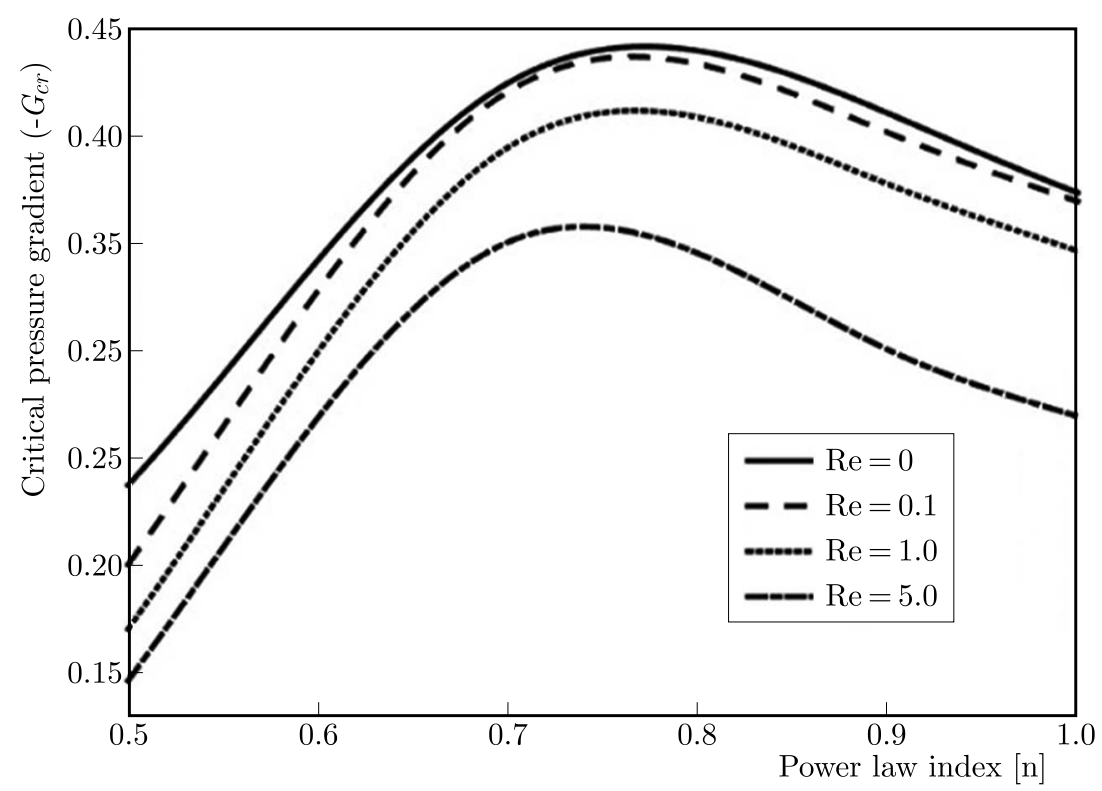

Fig. 3. Effect of the Reynolds number on the critical pressure gradient vs. the power-law index $n$ for different small Reynolds numbers for $T_{y}=0.2, \beta=1, H=40$, and $T=0.5$

Figure 4 shows the variation of the critical pressure gradient versus the yield stress for four Reynolds numbers. As it can be seen in this figure, increasing the Reynolds number has a destabilizing effect on the flow. This figure also shows that at a given Reynolds number, increasing the yield stress decreases the critical pressure gradient, meaning that the flow becomes unstable with an increase in the yield stress. Based on this figure, at any given Reynolds number, as the yield stress increases, the fluid becomes more and more unstable, which is a positive effect regarding the mixing process. Figure 4 also shows that, as the yield stress decreases, higher Reynolds numbers show higher instability.

Figure 5 shows the critical pressure gradient variation versus the Reynolds number for different power-law indices including both the shear-thinning and shear-thickening fluids. It can be seen in this figure that the inertia terms have a destabilizing effect on both the shear-thinning and shear-thickening fluids. From this figure, it can be concluded that for the Reynolds numbers 


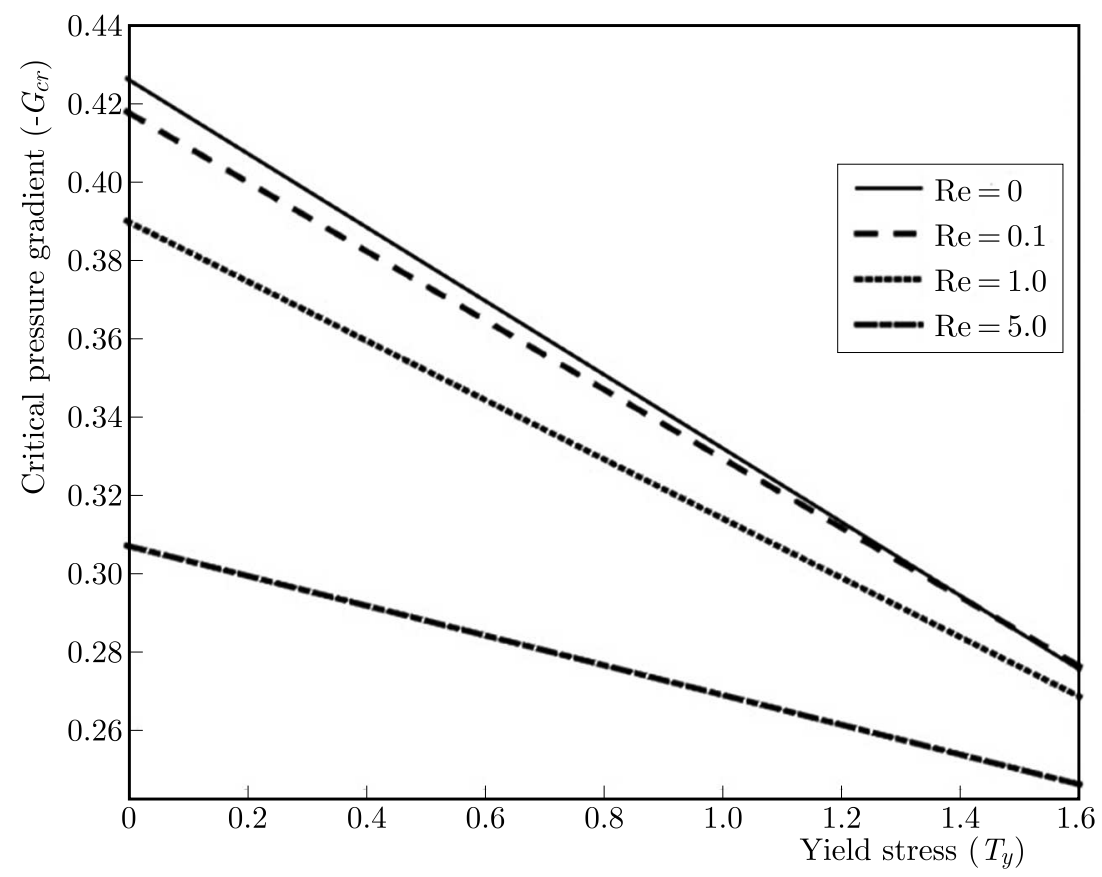

Fig. 4. Critical pressure gradient variation vs. the yield stress for different small Reynolds numbers for $H=40, T=0.5, \beta=1$, and $n=0.9$

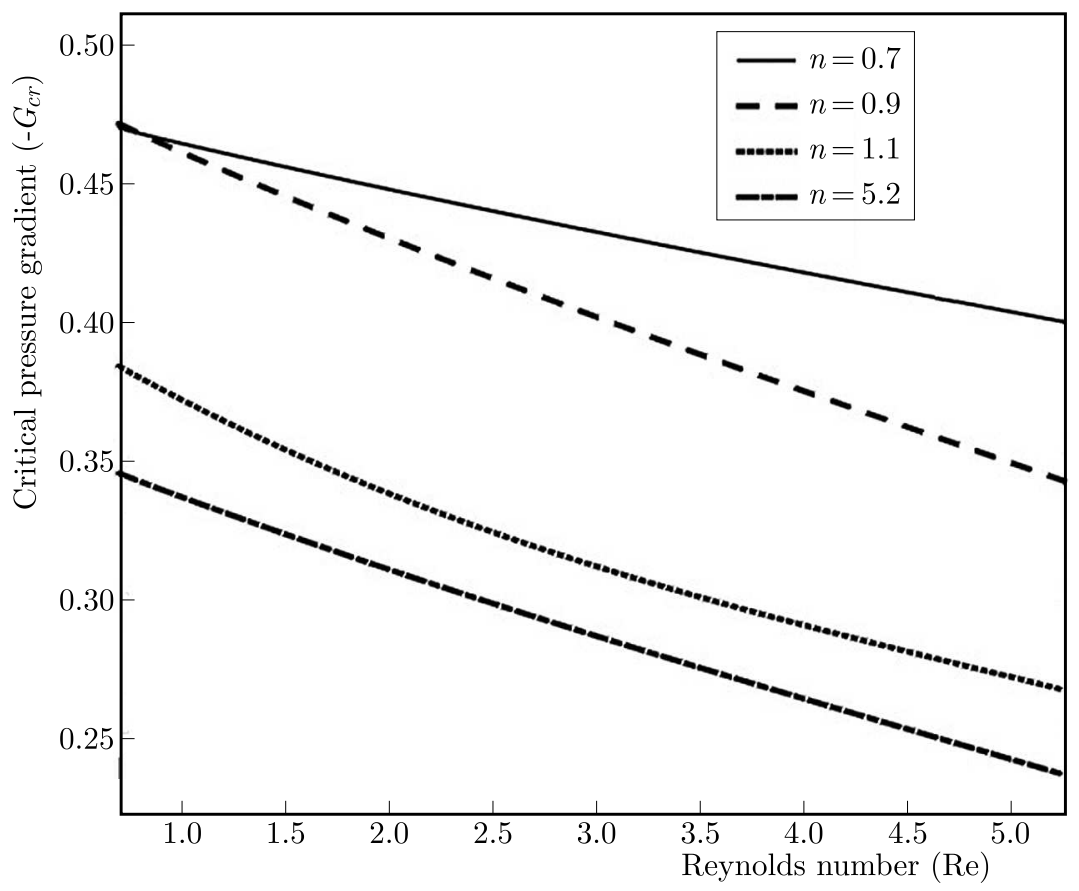

Fig. 5. Critical pressure gradient variation vs. the Reynolds number for $H=40, T_{y}=0.5, T=0.5$, and $\beta=1$

considered here, the shear-thinning fluids are the most stable ones as they need the largest critical pressure gradient to become unstable. Based on this figure, for both the shear-thinning $(n<1)$ and shear-thickening $(n>1)$ fluids, instability increases as the Reynolds number increases. This result is actually in line with the other results shown in the previous figures.

Figure 6 shows the effect of the yield stress on the critical pressure gradient for a coating obeying the Mooney-Rivlin model. The elastic parameter is unity and $\mathrm{Re}=5$. Based on the trend of the plots in Fig. 6, in the presence of inertia, the increase of the yield stress has a 
destabilizing effect on the non-zero Reynolds numbers flow. The figure also shows that as the power-law index increases, the fluid becomes more and more stable up to $n \simeq 0.75$, after which the fluid becomes unstable with a very low slope. This figure actually shows how the powerlaw exponent plays the key role in the hydroelastic instability phenomenon. To see the effect of this key role here, note that a decrease of the fluid viscosity is due to an increase of the shear rate, and noting that the fluid viscosity is actually controlled by the power-law index, then, the shear stress at the fluid-solid interface is also controlled by the index $n$. Therefore, the diffusive transfer of energy from the basic flow to the imposed perturbations is increased when the power-law index is decreased.

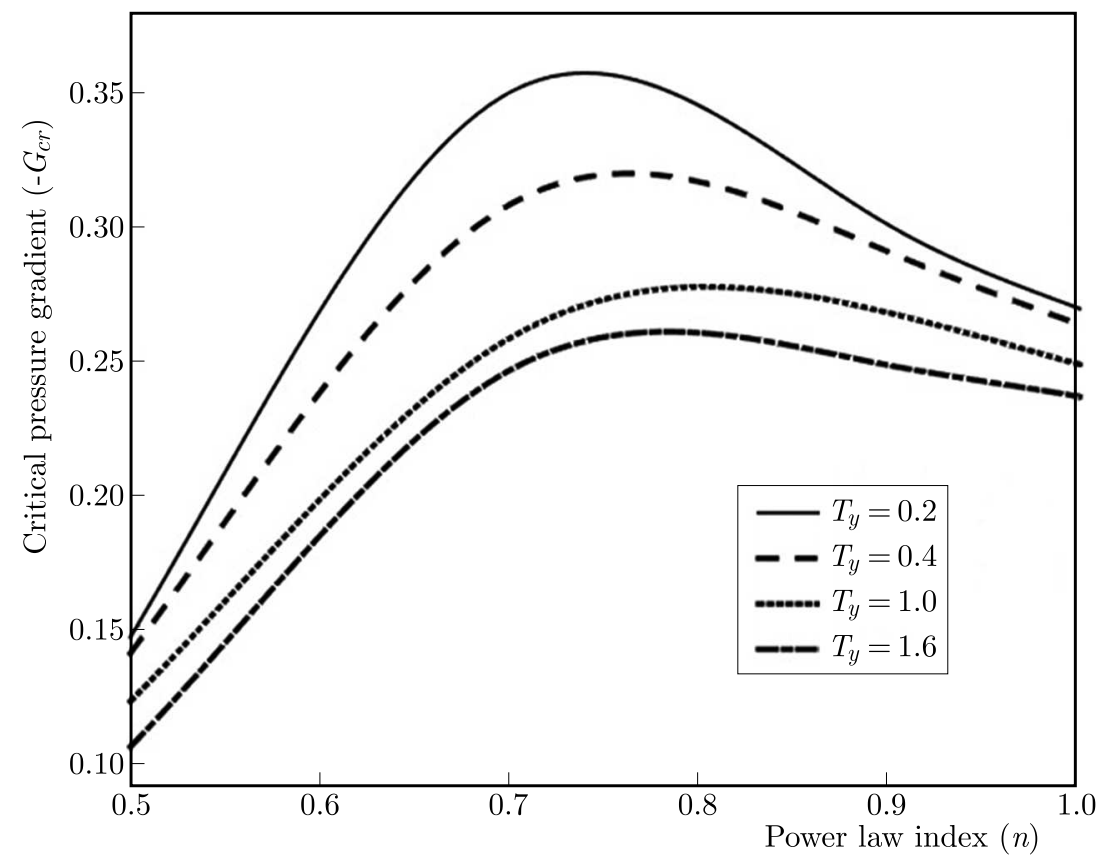

Fig. 6. Critical pressure gradient variation vs. the power-law index $n$ for $H=40, \beta=1, T=0.5$, and $\operatorname{Re}=5$

Figure 7 shows the critical pressure gradient variation vs. the power-law index for different elastic parameters $\beta$. This figure shows that the neo-Hookean solid $(\beta=0)$ is the least stable model regardless of the magnitude of the index $n$. Moreover, based on the figure, with an increase in the elastic parameter, the curves are shifted to more stable states. It is also noticed in this figure that in the presence of the inertia terms, for any given elastic parameter, the fluid shearthinning can have a stabilizing or destabilizing effect on the flow depending on the magnitude of the index $n$. An important effect of $\beta$ is that this parameter controls the first-normal-stressdifference at the fluid-solid interface. This parameter also affects the pressure discontinuity at the interface. Both effects, however, compete with the fluid shear stress (which initiates the instability) in affecting the hydroelastic instability, thereby, influencing the overall instability trend, as it can be seen in Fig. 7.

\section{Conclusions}

The aim of the present study has been to investigate the effects of inertia on the stability of the Poiseuille flow of the Herschel-Bulkley fluids passing through a two-dimensional channel. The channel is assumed to be coated with a hyperelastic polymeric material which obeys the two-constant Mooney-Rivlin model. This study is actually an extension of the authors' previous work in which the inertia terms were excluded from the governing equations, hence the effect of 


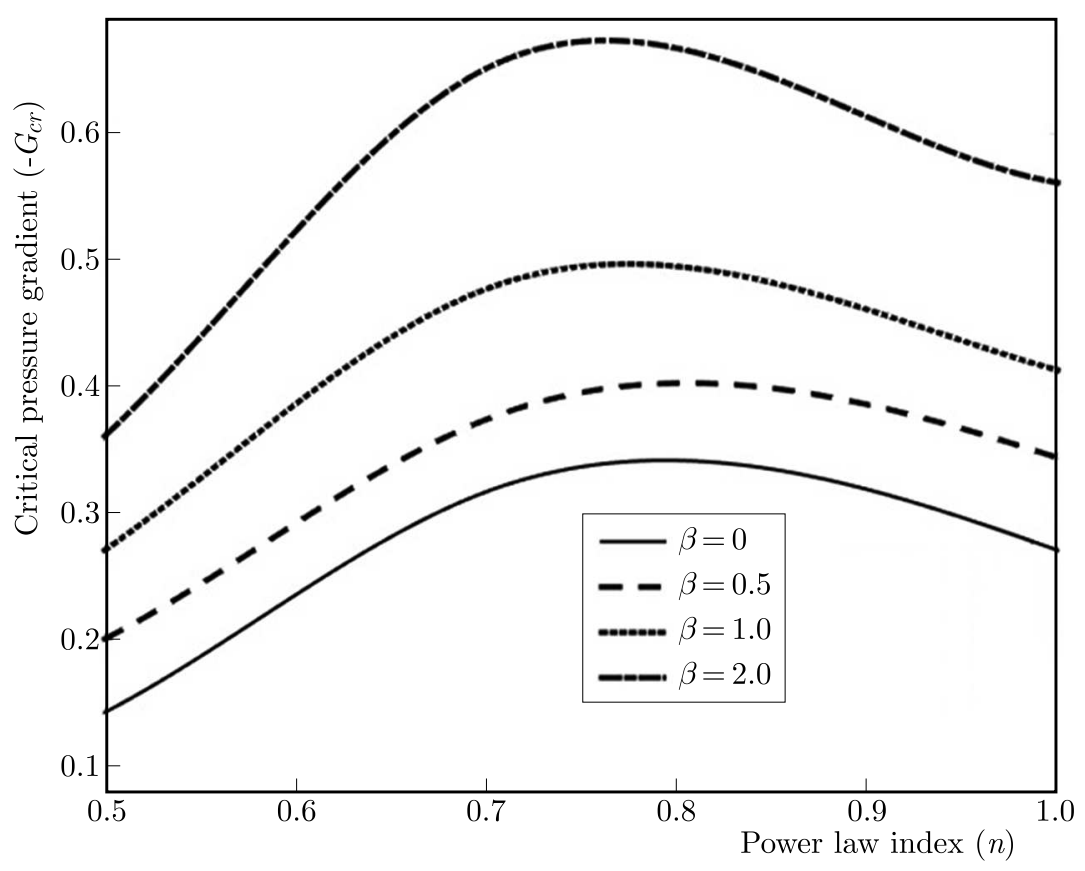

Fig. 7. Critical pressure gradient variation vs. the power-law index for $H=40, T_{y}=0.5, T=0.5$, and $\operatorname{Re}=1$

inertia on the fluid flow stability was never determined. In addition, here, unlike the previous works, the effect of the power-law index $n$ on both the shear-thinning and shear-thickening fluids flow are investigated. The above two new contributions in this work enable the results of this study not only to embrace a larger group of fluids, but also to be more realistic and reliable than ever before.

After determining appropriate governing equations for both the polymeric coating and the non-Newtonian fluid used, the base-state solutions have been obtained. Next, the solutions were subjected to infinitesimally-small disturbances after which the non-linear terms were eliminated. The equations after being reduced to two forth-order ordinary differential equations were solved numerically. A shooting technique numerical code was developed to solve (after some lengthy mathematical manupulations) the two fourth-order ordinary differential equations with appropriate boundary conditions. Having solved these equations numerically, the effects of the Reynolds number, yield stress and the elastic parameter on the growth rate of the unstable modes were investigated.

Based on the obtained results, it has been found that the inertia, in general, played a destabilizing role in the flowing fluids. It was determined that by increasing the Reynolds number, the flow became more unstable regardless of the yield stress values. It was also found that considering inertia in the governing equations did not affect the yield stress. Also, as the results showed, for extremely shear-thinning fluids, the yield stress was slightly stabilizing the flow, and for a wide range of power-law indices (close to unity), the yield stress was predicted to have a destabilizing effect on the pressure-driven flow in channels lined with the compliant coating. Based on the numerical results obtained in this work, it was noted that the elastic parameter strongly affected the hydroelastic stability. That is, Mooney-Rivlin coating became more stable than the neo-Hookean coating regardless of the values of the Reynolds number and the yield stress. The above findings of this study now help the investigators in the field to determine how to optimize the mixing efficiency when dealing with the hydroelastic instability of non-Newtonian fluids flowing in channels lined with polymeric gels. The more realistic and meaningful results of this work will definitely affect the microfluidic field research activities. 


\section{References}

1. Babenko V.V., Kozlov L.F., 1972, Experimental investigation of hydrodynamic stability on rigid and elastic damping surfaces, Journal of Hydraulic Research, 10, 383-408

2. Bird R.B., Armstrong R.C., Hassager O., 1987, Dynamics of Polymeric Liquids, 1, John Wiley, New York

3. Chien W.L., Rising H., Ottino J.M., 1986, Laminar and chaotic mixing in several cavity flows, Journal of Fluid Mechanics, 170, 355-377

4. Davies C., Carpenter P.W., 1997, Instabilities in a plane channel flow between compliant walls, Journal of Fluid Mechanics, 352, 205-243

5. Drazin P.G., Reid W.H., 2004, Hydrodynamic Stability, 2nd edit., Cambridge University Press

6. Franjione J.G., Ottino J.M., 1992, Symmetry concepts for the geometric analysis of mixing flows, Philosophical Transactions of the Royal Society A, 338, 301-323

7. Fu T.S., Joseph D.D., 1970, Linear stability of asymmetric flow in channels, Physics of Fluids, 13, $217-222$

8. GaD-EL-HaK M., 2002, Compliant coatings for drag reduction, Progress in Aerospace Sciences, 38, 77-99

9. Gkanis V., Kumar S., 2005, Stability of pressure-driven creeping flows in channels lined with a nonlinear elastic solid, Journal of Fluid Mechanics, 524, 357-375

10. Jafargholinejad S., 2015, Hydroelastic instability of Herschel-Bulkley fluids in channel flows, Ph.D. dissertation, Islamic Azad University

11. Jafargholinejad S., Najafi M., Sadeghy K., 2015, Hydroelastic instability of viscoplastic fluids in planar channel flow, Journal of the Society of Rheology, Japan, 43, 5, 157-164

12. Jensen K.F., 1999, Micromechanical systems: status, challenges and opportunities, AIChE Journal, 45, 2051-2054

13. Kramer M.O., 1960, Boundary-layer stabilization by distributed damping, Journal of the Aerospace Sciences, 27, 1, 69-69

14. Kramer M.O., 1960, Boundary layer stabilization by distributing damping, Journal of the American Society for Naval Engineers, 72, 25-33

15. Kandlikar S.G., Willistein D.A., Borrelli J., 2005, Experimental evaluation of pressure drop elements and fabricated nucleation sites for stabilizing flow boiling in microchannels, Third International Conference on Microchannels and Minichannels, ASME Paper, ICMM2005-75197, Toronto, Canada

16. Lai W.M., Rubin D., Krempl E., 2010, Introduction to Continuum Mechanics, 4th Ed., Elsevier

17. Lee K.C., Finlayson B.A., 1986, Stability of plane Poiseuille and Couette flow of a Maxwell fluid, Journal of Non-Newtonian Fluid Mechanics, 21, 1, 65-78

18. Muralikrishnan R., Kumaran V., 2002, Experimental study of the instability of the viscous flow past a flexible surface, Physics of Fluids, 14, 2, 775-780

19. Ottino J.M., 1989, The Kinematics of Mixing: Stretching, Chaos, and Transport, Cambridge University Press

20. Pourjafar M., Hamedi H., Sadeghy K., 2015, Stability of power-law fluids in creeping plane Poiseuille: the effect of wall compliance, Journal of Non-Newtonian Fluid Mechanics, 216, 22-30 